\title{
Semiempirical Correlation between P-Wave Velocity and Thermal Conductivity of Frozen Silty Clay Soil
}

\author{
Yadong Ji, ${ }^{1,2}$ Kaipeng Zhu, ${ }^{1,2}$ Chao Lyu $\left(\mathbb{D},{ }^{3}\right.$ Shidong Wang, ${ }^{1,2}$ Dianyan Ning, ${ }^{1,2,4}$ Juan Fan, ${ }^{1,2}$ \\ and Lei Shi ${ }^{1,2}$ \\ ${ }^{1}$ Xi'an Research Institute Co. Ltd., China Coal Technology and Engineering Group Corp., Xi'an, 710077, Shaanxi, China \\ ${ }^{2}$ Shaanxi Key Laboratory of Coal Mine Water Hazard Prevention and Control Technology, Xi'an, 710077, Shaanxi, China \\ ${ }^{3}$ School of Resources and Geosciences, China University of Mining and Technology, Xuzhou 221116, China \\ ${ }^{4}$ School of Geology and Environment, Xi'an University of Science and Technology, Xi'an 710054, China
}

Correspondence should be addressed to Chao Lyu; lvchao@cumt.edu.cn

Received 6 February 2021; Revised 13 March 2021; Accepted 30 March 2021; Published 12 April 2021

Academic Editor: Isabelle Sochet

Copyright (C) 2021 Yadong Ji et al. This is an open access article distributed under the Creative Commons Attribution License, which permits unrestricted use, distribution, and reproduction in any medium, provided the original work is properly cited.

In this study, the thermal conductivity and P-wave velocity of silty clay soil with different water contents are investigated through experiments at different temperatures, and a theoretical correlation between thermal conductivity and wave velocity is established. With temperature decline, the unfrozen water content is reduced and frost heave cracks propagate in soil samples. The variations in thermal conductivity and P-wave velocity are summarized as four phases. The freezing temperature of silty clay soil is between $-2^{\circ} \mathrm{C}$ and $-4^{\circ} \mathrm{C}$. There is an inversely proportional relationship between thermal conductivity and P-wave velocity for silty clay soil at temperatures below freezing. The experimental results show that the theoretical correlation can well explain the relationship between P-wave velocity and thermal conductivity. These findings provide a possibility for determining the thermal conductivity easily and quickly in geothermal systems and underground engineering projects.

\section{Introduction}

Seasonal frozen regions are widely distributed in the civilized world. The structure, physical and mechanical properties, and thermal physical properties of the soil undergo substantial changes due to the freeze-thaw cycle caused by temperature changes throughout the year [1]. In addition, the thawing temperature decreases as the higher pressure is applied on the ice or soil. The thawing of the seasonal frozen soil in the cold regions occurs because of the substructures and superstructures, which are contacting the frozen soil. Subsequently, the microfissures can be generated due to the volume shrinkage of frozen soil. The bearing capacity of foundation decreases, and the consolidation settlement of the superstructures constructed in the cold regions occurs accordingly. When the engineering structure is built on the seasonally frozen soil, the interaction between engineering structure and frozen soil is therefore studied deeply. Especially, the temperature field, frost heave, and consolidation and settlement of frozen soil need to be studied in depth $[2,3]$. Soil thermal conductivity is an important heat transfer property which shows the ability of soil to conduct heat $[4,5]$. There are many factors that affect the thermal conductivity such as mineral composition, moisture content, porosity, and temperature. In addition, thermal conductivity is an important parameter in the calculation of the temperature field $[6,7]$. The analysis and evaluation of soil thermal conductivity during the freeze-thaw cycle have become a focus in engineering and theoretical research. The estimated temperature distribution and freezing degree are essential to the structural health of buildings [8].

In the last few decades, some experimental and theoretical approaches have been proposed in the literature to determine the soil thermal conductivity [9-13]. For example, the weighted average thermal conductivity of each component in the soil matrix was proposed in the De Vries 
model [14] and a correlation between thermal conductivity and degree of saturation which incorporates a variable kappa that accounts for the soil type effect was deduced by Cote and Konrad [15]. Lu et al. [16] carried out a series of tests with a thermo-time domain reflectometry probe on twelve types of natural soils which ranged from sand and silt to clay loam and established a thermal conductivity model by examining a new relationship between thermal conductivity and saturation. Ultrasonic technique has been used to characterize the constituents and structure of soils [17]. For instance, researchers used the ultrasonic technique to determine physical and mechanical properties of frozen soils [18]. The main factors affecting soil P-wave velocity are soil mineralogy, saturation, porosity, cementation, and temperature. The fact that both thermal conductivity and $\mathrm{P}$-wave velocity are affected by the same parameters may help to determine their correlation $[13,19]$. In addition, the elastic waves of soil can be determined easily and quickly, and therefore determining the soil thermal conductivity from soil elastic wave is possible and timely. However, very few studies have attempted to generalize the correlation between thermal conductivity and elastic wave $[20,21]$.

In this study, to thoroughly examine the variations in thermal conductivity and P-wave velocity of silty clay soil, experiments on soil samples with different water contents at different temperatures are conducted and an association model for these two physical parameters is established. The applicability of the proposed theoretical equations for thermal conductivity and P-wave velocity of silty clay soil is also validated.

\section{A Theoretical Model for P-Wave Velocity and Thermal Conductivity of Frozen Soil}

Few research studies have attempted to develop theoretical models to determine the thermal conductivity and P-wave velocity of frozen soils. Moreover, generalizations of the relationships between thermal conductivity and P-wave velocity of frozen soils have yet to be derived. The thermal conductivity of frozen soil depends on the thermal conductivity of the single component (solid particles, water, ice, and air) and upon their volume fractions. According to the contributions of four-phase substances, the series model simplifies the structure of soils [22]. If the direction of the heat flow is parallel to the direction of each component (Figure 1), the thermal conductivity of frozen soils can be determined by the weighted arithmetic mean method as follows:

$$
\lambda=\sum \lambda_{i} S_{i}=\lambda_{s} S_{s}+\lambda_{w} S_{w}+\lambda_{i} S_{i}+\lambda_{a} S_{a},
$$

where $\lambda$ and $S$ are the thermal conductivity and cross section area of frozen soil, respectively, and the subscripts $s, w$, $i$, and a represent solid particles, pore water, ice, and air, respectively $\left(S_{\mathrm{s}}+S_{\mathrm{w}+} S_{\mathrm{i}+} S_{\mathrm{a}}=1\right)$.

It is suggested that the change in volume of water when it is frozen can be neglected. The unfrozen water content of the frozen soil at a certain temperature is $w_{u}$. Then,

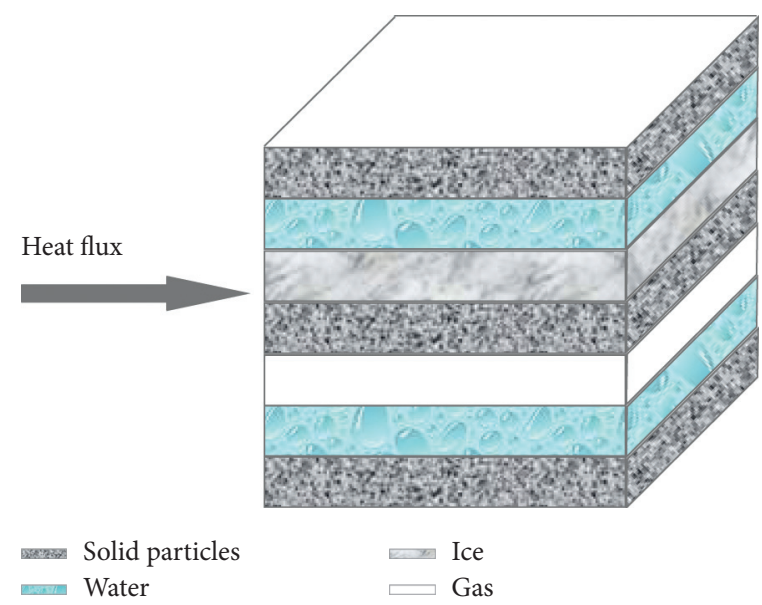

Figure 1: Parallel plate phase distribution in the series model.

$$
\begin{aligned}
\frac{S_{w}+S_{i}+S_{a}}{S} & =n, \\
\frac{S_{s}}{S} & =1-n, \\
\frac{S_{i}}{S} & =n S_{r}\left(1-w_{u} / w\right), \\
\frac{S_{w}}{S} & =\frac{n S_{r} w_{u}}{w},
\end{aligned}
$$

where $n, S_{\mathrm{r}}$, and $w$ are the porosity, saturation, and gravimetric water content of the frozen soil. Equation (1) can be described as

$$
\lambda=\lambda_{s}(1-n)+\frac{\lambda_{w} n S_{r} w_{u}}{w}+\lambda_{i} n S_{r}\left(1-\frac{w_{u}}{w}\right)+\lambda_{a} n\left(1-S_{r}\right) .
$$

Since $n\left(1-S_{\mathrm{r}}\right)<1$ and $\lambda_{\mathrm{a}} \ll \lambda_{s}, \quad \lambda_{a} n\left(1-S_{r}\right) \quad$ can be neglected. Equation (3) can be written as

$$
\lambda=\lambda_{s}(1-n)+n S_{r}\left[\frac{\left(\lambda_{w}-\lambda_{i}\right) w_{\mathrm{u}}}{w+\lambda_{i}}\right] .
$$

The porosity and saturation are constants for a soil with a given water content, and $\lambda_{s}, \lambda_{w}$, and $\lambda_{i}$ are constants. Thus, equation (4) can be described as

$$
\lambda=a+\frac{b w_{\mathrm{u}}}{w},
$$

where $a=\lambda_{s}(1-n)+n S_{r} \lambda_{i}$ and $b=n S_{r}\left(\lambda_{w}-\lambda_{i}\right)$ are constants. Previous studies have demonstrated that the wave velocity can be used to determine the unfrozen water content $[23,24]$. Timur [25] proposed a model to estimate the wave velocity in a frozen soil, which is written as

$$
\frac{1}{v}=\frac{\phi_{w}}{v_{w}}+\frac{\phi_{i}}{v_{i}}+\frac{\phi_{s}}{v_{s}},
$$

where $v, v_{s}, v_{w}$, and $v_{i}$ are the effective, solid, water, and ice wave velocities and $\phi_{w}, \phi_{i}$, and $\phi_{s}$ are the proportion of the phases $\left(\phi_{w}+\phi_{i}+\phi_{s}=1\right)$. Then, 


$$
\begin{array}{r}
\phi_{w}=n S_{r} \frac{w_{u}}{w}, \\
\phi_{i}=n S_{r}\left(1-\frac{w_{u}}{w}\right), \\
\phi_{s}=1-n .
\end{array}
$$

Equation (6) can be described as

$$
\frac{1}{v}=n S_{r}\left[\frac{w_{u}}{w}\left(\frac{1}{v_{w}}-\frac{1}{v_{i}}\right)+1\right]+\frac{1-n}{v_{s}} .
$$

Since $v_{s}, v_{w}$, and $v_{i}$ are constants, equation (7) can be described as

$$
\frac{1}{v}=\frac{p w_{\mathrm{u}}}{w}+q
$$

where $p=n S_{r}\left(1 / v_{w}-1 / v_{i}\right)$ and $q=n S_{r}+\left((1-n) / v_{s}\right)$ are constants for a soil with a given water content. Therefore, the unfrozen water content can be described as

$$
\frac{w_{u}}{w}=\frac{1}{p}\left(\frac{1}{v}-q\right) .
$$

Incorporating equation (10) into equation (5), the correlation between thermal conductivity $(\lambda)$ and wave velocity $(v)$ is expressed as

$$
\lambda=A+\frac{B}{v}
$$

where $A=\lambda_{s}(1-n)+n S_{r} \lambda_{i}-\left(\lambda_{w}-\lambda_{i}\right)\left(\left(n S_{r}+\left((1-n) / v_{s}\right)\right.\right.$ )$\left./\left(1 / v_{w}-1 / v_{i}\right)\right)$ and $B=\left(\lambda_{w}-\lambda_{i}\right) /\left(1 / v_{w}-1 / v_{i}\right)$ are related to the soil porosity and soil saturation and are constants for a soil with a given water content. It can be therefore observed that the thermal conductivity of frozen soil is inversely proportional to wave velocity.

\section{Materials and Methods}

3.1. Preparation of Soil Samples. The silty clay soil used for the determination of thermal conductivity and P-wave velocity was collected from typical eluvial deposits in farmland in Xuzhou city, Jiangsu Province, China. The basic physical properties and the grain-size distribution of the experimental silty clay soil are shown in Table 1 and Figure 2. The main composition is quartz and kaolinite. First, the soil samples with different gravimetric water contents of $10 \%$, $12 \%, 15 \%, 18 \%$, and $20 \%$ were put in a stainless steel cylindrical container $(\Phi 5 \times 10 \mathrm{~cm})$. The detailed production process of soil samples is given by Geng et al. [26]. After compaction, the samples are cured naturally for $24 \mathrm{~h}$ and then demoulded. Then, the soil samples were subjected to eleven target temperatures of $10^{\circ} \mathrm{C}, 5^{\circ} \mathrm{C}, 2^{\circ} \mathrm{C}, 0^{\circ} \mathrm{C},-2^{\circ} \mathrm{C},-4^{\circ} \mathrm{C}$, $-6^{\circ} \mathrm{C},-8^{\circ} \mathrm{C},-10^{\circ} \mathrm{C},-15^{\circ} \mathrm{C}$, and $-20^{\circ} \mathrm{C}$ in a $\mathrm{BPH}-\mathrm{B}$ incubator (Shanghai Yiheng Scientific Instruments Co., Ltd, China) for $24 \mathrm{~h}$ to ensure uniform cooling after reaching the target temperatures. The thermal conductivity and $\mathrm{P}$-wave velocity were then measured.
3.2. Test Instruments and Method. The thermal conductivity of soil samples was measured through the test hole on the incubator using a DRE-2C thermal conductivity tester which uses the transient plane source method. The details on the DRE-2C thermal conductivity tester are given in the study by Sun and Lü [13]. Then, the P-wave velocity was measured using a RSM-SY5 acoustic wave detector (see Figure 3 ). The sampling interval was $0.5 \mu \mathrm{s}$. Vaseline petroleum jelly was used as a medium to maximize acoustic coupling and smeared onto the transducer and the surface of the sample.

\section{Test Results and Analysis}

4.1. Analysis of P-Wave Velocity. When a soil sample is subjected to a low temperature, the phase transition of water and the development of cracks will lead to changes in the number of pores and pore structure, which will change their $\mathrm{P}$-wave velocities. The freezing temperature of soils is affected by the soil texture, structure, moisture, and salinity. Ultrasonic p-wave velocity has been used in previous studies to determine the unfrozen water content and freezing temperature of frozen soil $[23,24]$. The temperature at which the $\mathrm{P}$-wave velocity rapidly increases can be considered as the freezing temperature. The variations in the P-wave velocity of the samples with different water contents are shown in Figure 4. It can be observed that the freezing temperature of the silty clay samples is about $-2^{\circ} \mathrm{C}$. At a temperature range of $10^{\circ} \mathrm{C}$ to $-2^{\circ} \mathrm{C}$ or Stage $\mathrm{I}$, the $\mathrm{P}$-wave velocity is almost constant. The $\mathrm{P}$-wave velocity of the samples with a water content of $10 \%$ is about $750 \mathrm{~m} / \mathrm{s}$ while the P-wave velocity of those with a water content of $15 \%$ and $18 \%$ is close to $1000 \mathrm{~m} / \mathrm{s}$. When the temperature is low and ranges from $-2^{\circ} \mathrm{C}$ to $-20^{\circ} \mathrm{C}$, the $\mathrm{P}$-wave velocity varies and can be described as a rapid increase followed by a slower increase and then gradually becomes constant. The specific rates of increase are shown in Table 2. At a temperature range of $-2^{\circ} \mathrm{C}$ to $-6^{\circ} \mathrm{C}$ or Stage II, it can be seen that samples with a higher water content show a greater increase in their P-wave velocity. However, samples with a low water content show a greater increase in their $\mathrm{P}$-wave velocity from $-6^{\circ} \mathrm{C}$ to $-20^{\circ} \mathrm{C}$ or at Stages III and IV.

4.2. Analysis of Thermal Conductivity. Figure 5 shows the relationship between thermal conductivity and temperature of the silty clay samples with different water contents. It can be seen that the variations in thermal conductivity are divided into four stages. The temperature ranges from $10^{\circ} \mathrm{C}$ to $-2^{\circ} \mathrm{C}$ in Stage I, in which the thermal conductivity of samples with a water content of $10 \%, 12 \%, 15 \%, 18 \%$, and $20 \%$ is basically constant at 1.37, 1.45, 1.69, 1.68, and $1.66 \mathrm{~W} \cdot \mathrm{m}^{-1} \mathrm{~K}^{-1}$, respectively. At a temperature range of $-2^{\circ} \mathrm{C}$ to $-6^{\circ} \mathrm{C}$ or at Stage II, the thermal conductivity substantially increases with reduced temperature. However, the thermal conductivity quickly decreases as the temperature declines for the soil samples with a high water content (15 20\%) from $-4^{\circ} \mathrm{C}$ to $-6^{\circ} \mathrm{C}$. At a temperature range of $-6^{\circ} \mathrm{C}$ to $-10^{\circ} \mathrm{C}$ or Stage III, the thermal conductivity of all of the samples shows a slow trend of decline with decreased temperature. 
TABLE 1: Basic physical properties of experimental silty clay.

\begin{tabular}{lccccc}
\hline Water content (\%) & Natural density $\left(\mathrm{g} / \mathrm{cm}^{3}\right)$ & Degree of saturation (\%) & Liquid limit (\%) & Plastic limit (\%) & Plasticity index \\
\hline 25.06 & 1.76 & 72.8 & 29 & 18.5 & 10.5 \\
\hline
\end{tabular}

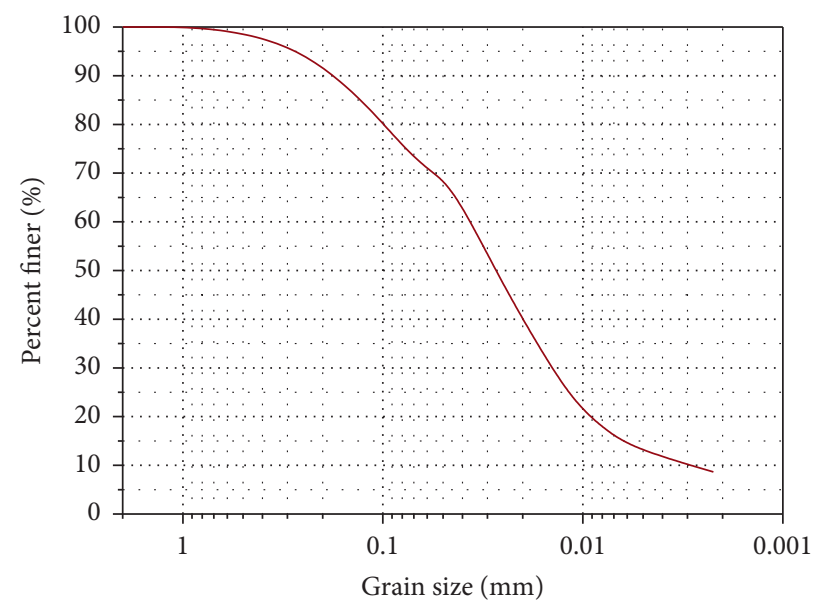

Figure 2: Plot of grain-size distribution.

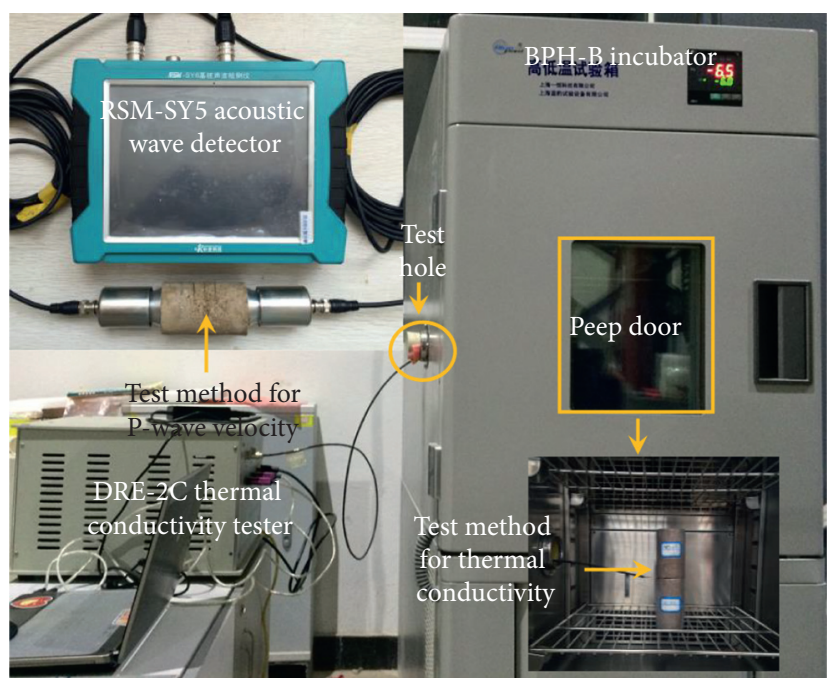

FIGURE 3: Experimental set up for measuring the thermal conductivity and P-wave velocity.

When the temperature is lower than $-10^{\circ} \mathrm{C}$ or at Stage IV, the thermal conductivity remains constant on the whole at $1.84,1.95,2.1,2.15$, and $2.25 \mathrm{~W} \cdot \mathrm{m}^{-1} \mathrm{~K}^{-1}$ with a water content of $10 \%, 12 \%, 15 \%, 18 \%$, and $20 \%$, respectively. It is obvious that the thermal conductivity at $-20^{\circ} \mathrm{C}$ is about $0.5 \mathrm{~W} \cdot \mathrm{m}^{-1} \mathrm{~K}^{-1}$ higher than that at $10^{\circ} \mathrm{C}$.

\section{Discussion}

The unfrozen water content in frozen soil has a great influence on the physical and mechanical properties of soil. Previous research studies have shown that the unfrozen water content is rapidly reduced from $-2^{\circ} \mathrm{C}$ to $-5^{\circ} \mathrm{C}$ and then is reduced slowly [27-29]. When the temperature is below $-20^{\circ} \mathrm{C}$, the unfrozen water content is approximately the same even though the initial water content is different. In our experiment, the unfrozen water content greatly varies between $-2^{\circ} \mathrm{C}$ and $-6^{\circ} \mathrm{C}$. The variations in the thermal conductivity and P-wave velocity can be examined as four phases: pretransformation, intense transformation, transition, and permanently frozen phases.

(1) Pretransformation (temperature range of $10^{\circ} \mathrm{C}$ to $-2^{\circ} \mathrm{C}$ ): The degree that the samples are frozen is very low, and there is no substantial change in their internal structure. The thermal conductivity decreases slowly with decrease in temperature due to the deceleration of molecular heat movement. The P-wave velocity is basically constant.

(2) Intense transformation (temperature range of $-2^{\circ} \mathrm{C}$ to $-6^{\circ} \mathrm{C}$ ): Most of water in samples is frozen, thus filling the pores and cementing the soil particles. The 


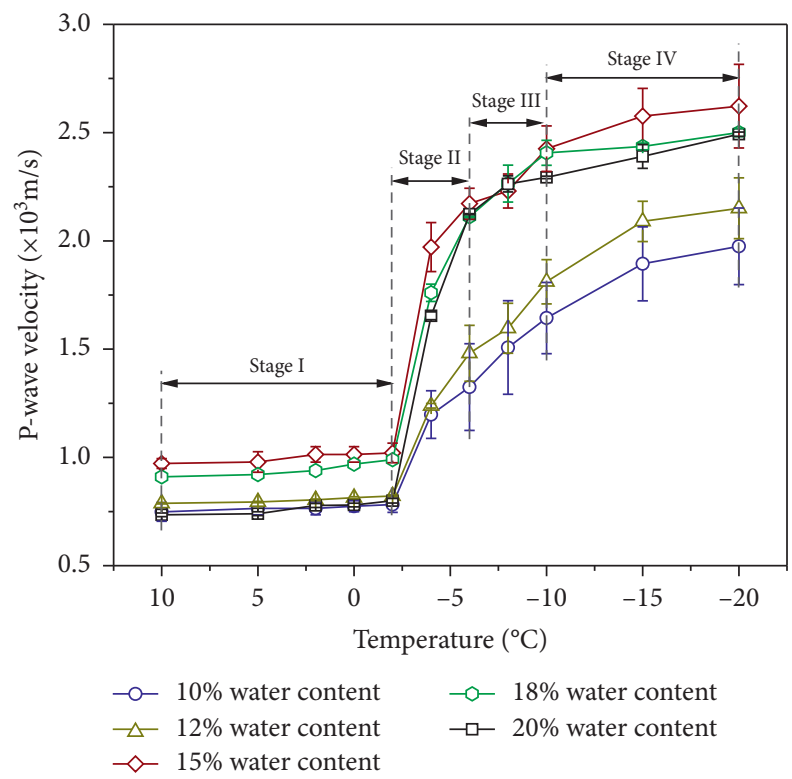

FIgURE 4: Variations in P-wave velocity with different water contents versus temperature.

TABLE 2: Rate of change in P-wave velocity per degree centigrade with different water contents at different stages $(\mathrm{m} / \mathrm{s})$.

\begin{tabular}{lccccc}
\hline \multirow{2}{*}{ Stage } & \multicolumn{5}{c}{ Water content } \\
& $10 \%$ & $12 \%$ & $15 \%$ & $18 \%$ & $20 \%$ \\
\hline II & 135.7 & 139.9 & 287.9 & 280.5 & 330.9 \\
III & 79.7 & 107.5 & 63.4 & 73.9 & 42.5 \\
IV & 57.9 & 84.8 & 49.1 & 23.4 & 49.9 \\
\hline
\end{tabular}

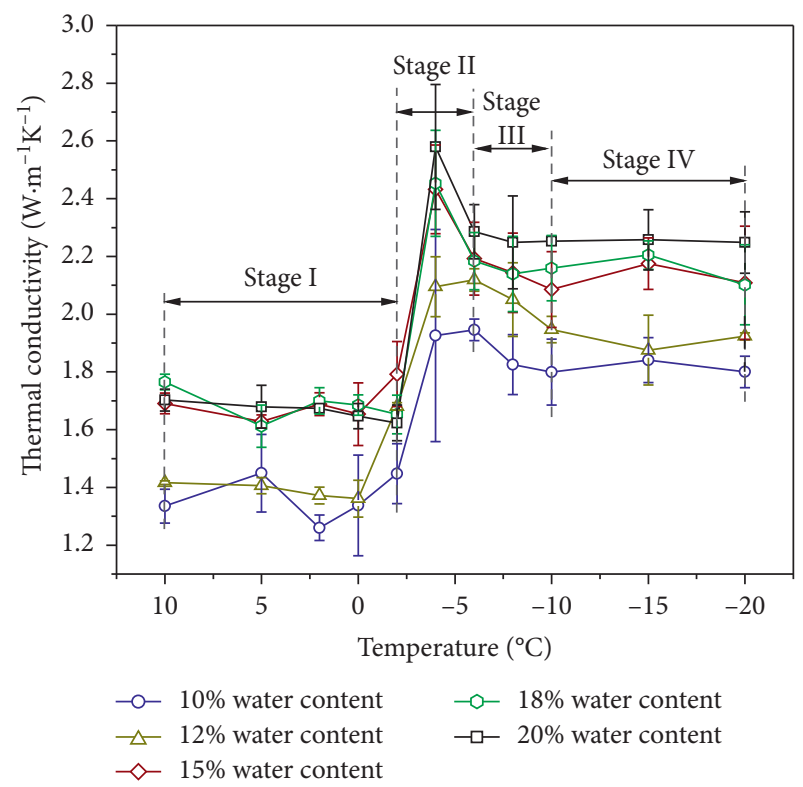

FIGURE 5: Variations in thermal conductivity of silty clay samples with different water contents versus temperature.

clay therefore changes from a particle assembly of individual grains to a solid composite that consists of particles and solid water [30]. Meanwhile, since the thermal conductivity and P-wave velocity of water is lower than ice, the thermal conductivity and P-wave velocity of the samples increase substantially. In addition, the volume expansion of water due to the phase transition separates the solid particles [24]. Cracks therefore propagate on the surface of the soil samples due to frost heave. At a temperature of $-6^{\circ} \mathrm{C}$, frost heave cracks are greatly generated for the samples with a higher water content as shown in Figure 6(a). Consequently, the thermal conductivity slightly reduces with decreased temperature. The thermal conductivity for the samples with a water content of $10 \%$ and $12 \%$ increases as there are few cracks. Soil samples with high water content have more frost heave cracks, so the volume fraction of air in samples is higher. Therefore, the P-wave velocity for the samples with a water content of $18 \%$ and $20 \%$ is lower than that for the samples with a water content of $15 \%$.

(3) Transition (temperature range of $-6^{\circ} \mathrm{C}$ to $-10^{\circ} \mathrm{C}$ ): A small amount of water continues to freeze in soil samples. Therefore, the thermal conductivity is slowly decreased with the development and extension of frost heave cracks as shown in Figure 6(b). The P-wave velocity is gradually increased, and the rate of increase in the $\mathrm{P}$-wave velocity is less than that in the intense transformation phase.

(4) Permanently frozen (temperature range of $-10^{\circ} \mathrm{C}$ to $-20^{\circ} \mathrm{C}$ ): In general, soil samples have a low unfrozen water content, and thus the frost heave cracks are rarely generated. The thermal conductivity remains constant, and the P-wave velocity is slightly increased.

Figure 7 shows the variations in the generalized correlation between thermal conductivity and P-wave 


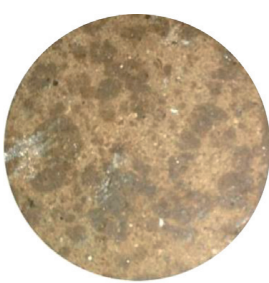

$10 \%$ water content

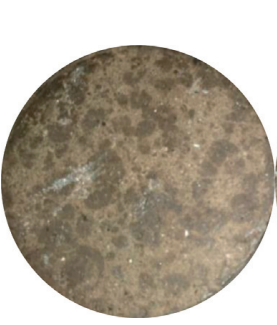

$10 \%$ water content

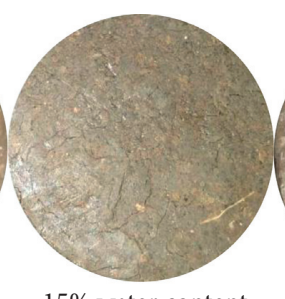

$15 \%$ water content

(a)

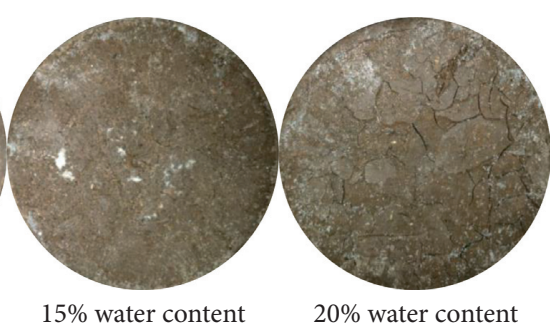

(b)

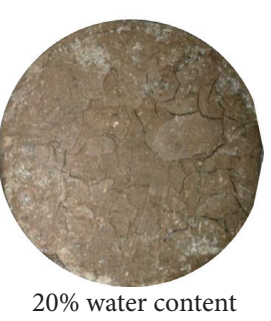

$20 \%$ water content
FIGURE 6: Frost heave cracks on samples with different water contents at different temperatures. (a) $-6^{\circ} \mathrm{C}$; (b) $-10^{\circ} \mathrm{C}$.

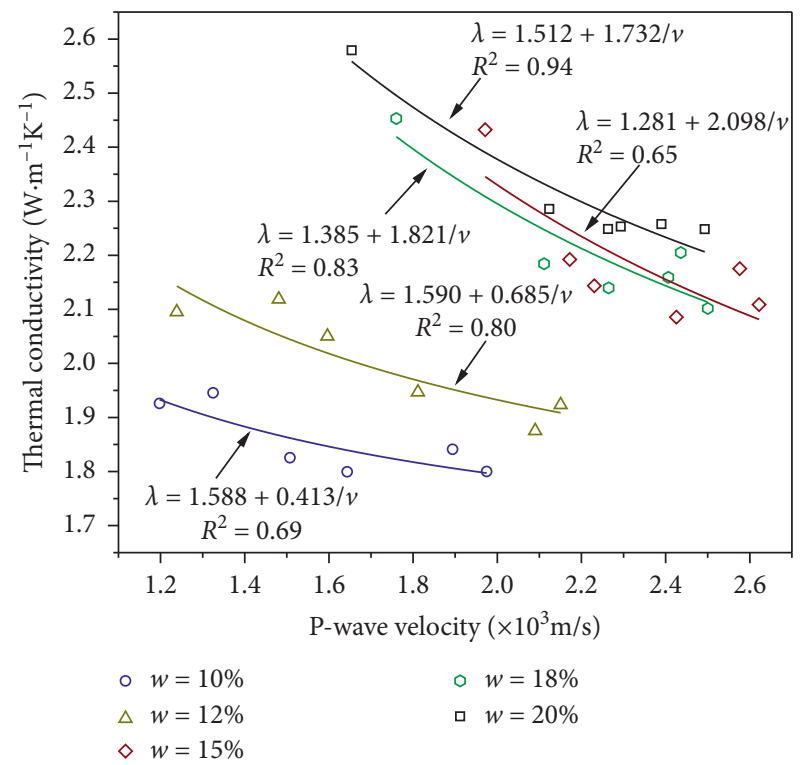

FIgURE 7: Relationship between thermal conductivity and P-wave velocity of frozen silty clay soil from $-4^{\circ} \mathrm{C}$ to $-20^{\circ} \mathrm{C}$.

velocity of frozen silty clay soil from $-4^{\circ} \mathrm{C}$ to $-20^{\circ} \mathrm{C}$. The thermal conductivity has an inversely proportional relationship with $\mathrm{P}$-wave velocity. The statistical parameters $A$ and $B$ for the various fits are listed in Table 3. The correlation coefficients of the equations fitted by the experimental data are high. The results of the experiments are consistent with the inversely proportional function of the theoretical correlation for the thermal conductivity and the $\mathrm{P}$-wave velocity. The parameters $A$ and $B$ are mainly related to the water content of samples. It can be observed from Table 3 that the parameter $A$ is first decreased with water content in general and then is
TABle 3: A summary of the statistical parameters $A$ and $B$ for the various fits.

\begin{tabular}{lccc}
\hline Initial water content $(\%)$ & $A$ & $B$ & $R^{2}$ \\
\hline 10 & 1.588 & 0.413 & 0.69 \\
12 & 1.590 & 0.685 & 0.80 \\
15 & 1.281 & 2.098 & 0.65 \\
18 & 1.385 & 1.821 & 0.83 \\
20 & 1.512 & 1.732 & 0.94 \\
\hline
\end{tabular}

increased when the water content exceeds $15 \%$. The fitting parameter $B$ is first increased with water content and then is decreased when the water content exceeds $15 \%$. Determining soil thermal conductivity through correlation to its $\mathrm{P}$-wave velocity seems to be a simple and effective solution. The generalized correlation between thermal conductivity and P-wave velocity for the frozen silty clay soil is studied in the paper. Future research needs to be conducted on the different types of soil, and more testing data need to be analyzed.

\section{Conclusions}

In summary, this paper has examined the variations in thermal conductivity and P-wave velocity for samples with different water contents under low temperatures based on the formation of frozen soil and analyzed the correlation between the various parameters. The main outcomes are summarized as follows:

(1) The variations in thermal conductivity and P-wave velocity with temperature can be summarized as four stages: pretransformation phase, intense transformation phase, transition phase, and permanently frozen phase. The thermal conductivity and P-wave velocity all rapidly increase in intense transformation phase due to the formation of ice which bonds the solid particles. The thermal conductivity of soil samples with a water content of $15 \%, 18 \%$, and $20 \%$ is slightly reduced at $-6^{\circ} \mathrm{C}$ due to the rapid growth of the frost heave cracks.

(2) The correlation between thermal conductivity and P-wave velocity of frozen silty clay soil is well described by the equation $\lambda=A+B / v$. The experimental results verify the validity of the theoretical equation for the thermal conductivity and the $\mathrm{P}$-wave velocity. The findings provide the possibility for estimating the thermal conductivity of frozen soil from the P-wave velocity in practice.

\section{Data Availability}

The data used to support the findings of this study are available from the corresponding author upon request.

\section{Conflicts of Interest}

The authors declare that they have no conflicts of interest. 


\section{Acknowledgments}

This research was supported by the Open Fund of Shaanxi Key Lab of Mine Water Hazard Prevention and Control (no. 6000200348).

\section{References}

[1] H. Jia, F. Zi, G. Yang et al., "Influence of pore water (ice) content on the strength and deformability of frozen argillaceous siltstone," Rock Mechanics and Rock Engineering, vol. 53, no. 2, pp. 967-974, 2020.

[2] G. H. Johnston, Permafrost: Engineering Design and Construction, pp. 77-127, J. Wiley, Hoboken, NJ, USA, 1981.

[3] Y. Ji, G. Zhou, and M. R. Hall, "Frost heave and frost heavinginduced pressure under various restraints and thermal gradients during the coupled thermal-hydro processes in freezing soil," Bulletin of Engineering Geology and the Environment, vol. 78, no. 5, pp. 3671-3683, 2019.

[4] S. Nikoosokhan, H. Nowamooz, and C. Chazallon, "Effect of dry density, soil texture and time-spatial variable water content on the soil thermal conductivity," Geomechanics and Geoengineering, vol. 11, no. 2, pp. 149-158, 2016.

[5] B. Ghauman and R. Lal, "Thermal conductivity, thermal diffusivity, and thermal capacity of some Nigerian soils," Soil Science, vol. 139, pp. 74-80, 1986.

[6] Y. M. Pang, X. H. Zhang, X. W. Guo, G. L. Xiao, and X. Q. Zhu, "Mesozoic and cenozoic tectono-thermal evolution modeling in the northern south yellow sea Basin," Chinese Journal of Geophysics, vol. 60, pp. 3177-3190, 2017.

[7] Y.-H. Zuo, B. Ye, W.-T. Wu et al., "Present temperature field and cenozoic thermal history in the dongpu depression, Bohai Bay Basin, North China," Marine and Petroleum Geology, vol. 88, pp. 696-711, 2017.

[8] W. Shan, Y. Liu, Z. Hu, and J. Xiao, "A model for the electrical resistivity of frozen soils and an experimental verification of the model," Cold Regions Science and Technology, vol. 119, pp. 75-83, 2015.

[9] T. Tokoro, T. Ishikawa, S. Shirai, and T. Nakamura, "Estimation methods for thermal conductivity of sandy soil with electrical characteristics," Soils and Foundations, vol. 56, no. 5, pp. 927-936, 2016.

[10] I. V. Nikolaev, W. H. Leong, and M. A. Rosen, "Experimental investigation of soil thermal conductivity over a wide temperature range," International Journal of Thermophysics, vol. 34, no. 6, pp. 1110-1129, 2013.

[11] N. Zhang and Z. Wang, "Review of soil thermal conductivity and predictive models," International Journal of Thermal Sciences, vol. 117, pp. 172-183, 2017.

[12] P. Samui, "Utilization of Gaussian process regression for determination of soil electrical resistivity," Geotechnical and Geological Engineering, vol. 32, no. 1, pp. 191-195, 2014.

[13] Q. Sun and C. Lü, "Semiempirical correlation between thermal conductivity and electrical resistivity for silt and silty clay soils," Geophysics, vol. 84, no. 3, pp. MR99-MR105, 2019.

[14] D. A. De Vries, "Thermal properties of soils," in Physics of the Plant Environment, W. R. Van Wijk, Ed., pp. 210-235, NorthHolland, Amsterdam, Netherlands, 1963.

[15] J. Côté and J. M. Konrad, "A generalized thermal conductivity model for soils and construction materials," Canadian Geotechnical Journal, vol. 42, no. 2, pp. 443-458, 2005.

[16] S. Lu, T. Ren, Y. Gong, and R. Horton, "An improved model for predicting soil thermal conductivity from water content at room temperature," Soil Science Society of America Journal, vol. 71, no. 1, pp. 8-14, 2007.

[17] Y. Nakano, R. J. Martin, and M. Smith, "Ultrasonic velocities of the dilatational and shear waves in frozen soils," Water Resources Research, vol. 8, no. 4, pp. 1024-1030, 1972.

[18] M. Christ and J.-B. Park, "Ultrasonic technique as tool for determining physical and mechanical properties of frozen soils," Cold Regions Science and Technology, vol. 58, no. 3, pp. 136-142, 2009.

[19] Q. Sun, C. Lyu, and W. Zhang, “The relationship between thermal conductivity and electrical resistivity of silty clay soil in the temperature range $-20^{\circ} \mathrm{C}$ to $10^{\circ} \mathrm{C}$," Heat and Mass Transfer, vol. 56, no. 6, pp. 2007-2013, 2020.

[20] T. Popp and H. Kern, "Thermal dehydration reactions characterised by combined measurements of electrical conductivity and elastic wave velocities," Earth and Planetary Science Letters, vol. 120, no. 1-2, pp. 43-57, 1993.

[21] M. Nouveau, G. Grandjean, P. Leroy, M. Philippe, E. Hedri, and H. Boukcim, "Electrical and thermal behavior of unsaturated soils: experimental results," Journal of Applied Geophysics, vol. 128, pp. 115-122, 2016.

[22] G. S. Jia, Z. Y. Tao, X. Z. Meng, C. F. Ma, J. C. Chai, and L. W. Jin, "Review of effective thermal conductivity models of rock-soil for geothermal energy applications," Geothermics, vol. 77, pp. 1-11, 2019.

[23] D.-Y. Wang, Y.-L. Zhu, W. Ma, and Y.-H. Niu, “Application of ultrasonic technology for physical-mechanical properties of frozen soils," Cold Regions Science and Technology, vol. 44, no. 1, pp. 12-19, 2006.

[24] M. Kang and J. S. Lee, "Evaluation of the freezing-thawing effect in sand-silt mixtures using elastic waves and electrical resistivity," Cold Regions Science and Technology, vol. 113, pp. 1-11, 2015.

[25] A. Timur, "Velocity of compressional waves in porous media at permafrost temperatures," Geophysics, vol. 33, no. 4, pp. 584-595, 1968.

[26] J. Geng and Q. Sun, "Effects of high temperature treatment on physical-thermal properties of clay," Thermochimica Acta, vol. 666, pp. 148-155, 2018.

[27] P. P. Overduin, D. L. Kane, and W. K. P. Van Loon, "Measuring thermal conductivity in freezing and thawing soil using the soil temperature response to heating," Cold Regions Science and Technology, vol. 45, no. 1, pp. 8-22, 2006.

[28] K. Watanabe and Y. Osada, "Simultaneous measurement of unfrozen water content and hydraulic conductivity of partially frozen soil near $0^{\circ} \mathrm{C}$," Cold Regions Science and Technology, vol. 142, pp. 79-84, 2017.

[29] L. Tang, K. Wang, L. Jin, G. Yang, H. Jia, and A. Taoum, "A resistivity model for testing unfrozen water content of frozen soil," Cold Regions Science and Technology, vol. 153, pp. 55-63, 2018.

[30] C. Lyu, Q. Sun, and W. Zhang, "Effects of $\mathrm{NaCl}$ concentration on thermal conductivity of clay with cooling," Bulletin of Engineering Geology and the Environment, vol. 79, no. 4, pp. 1449-1459, 2019. 